\title{
Radiofrequency catheter ablation for atrial flutter following orthotopic heart transplantation
}

\author{
M P I Pitt, R S Bonser, M J Griffith
}

\begin{abstract}
A 26 year old woman with a diagnosis of limb girdle muscular dystrophy and peripartum cardiomyopathy underwent orthotopic heart transplantation using standard atrial anastomoses. Recurrent atrial flutter was demonstrated in the absence of histological evidence of cellular rejection. Radiofrequency ablation of the flutter circuit was successfully performed allowing the avoidance of long term antiarrhythmic therapy. Ten weeks following ablation and cessation of amiodarone, presyncopal symptoms were found, associated with daytime and nocturnal sinus pauses of 2.9 seconds and 4.2 seconds, respectively. There was no evidence of AV node conduction impairment. In view of the continued absence of cellular rejection and the evident denervated state of the heart, the pauses reflected significant donor sinus node dysfunction; therefore, an AAIR permanent pacing system was implanted. At the time of pacemaker implantation atrial pacing at 150 beats/ min did not produce significant PR interval prolongation or AV block. This case serves to advise rigorous follow up in such patients to continue to seek evidence of sinus node dysfunction potentially requiring permanent pacemaker implantation. (Heart 1998;79:412-413)
\end{abstract}

Keywords: heart transplantation; atrial flutter; radiofrequency catheter ablation

Atrial flutter is often associated with the presence of underlying structural heart disease. While chemical and electrical cardioversion often result in the attainment of sinus rhythm, long term antiarrhythmic treatment is required in more than $50 \%$ of patients to maintain sinus rhythm. ${ }^{1}$ Electrophysiological study has allowed the mapping of atrial macro reentrant circuits in atrial flutter, thus facilitating attempts at radiofrequency ablation, with varying degrees of success. ${ }^{2}$

Atrial arrhythmias are common following orthotopic heart transplantation, with an incidence of $25-44 \% .^{34}$ Orthotopic heart transplantation employing bicaval anastomoses is associated with a lower frequency of atrial arrhythmias than standard atrial anastomoses. ${ }^{5}$ Atrial flutter, in particular, is frequently related to underlying myocardial rejection. We describe a patient with recurrent atrial flutter following orthotopic heart transplantation, successfully treated by ablation of the flutter pathway. The successful application of radiofrequency atrial flutter ablation has been reported only once previously following orthotopic heart transplantation. ${ }^{6}$ Ablation for atrial flutter has been successful in other patients with postoperative atrial scars. ${ }^{7}$

\section{Case report}

A 26 year old woman with a diagnosis of limb girdle muscular dystrophy and peripartum cardiomyopathy underwent orthotopic heart transplantation using standard atrial anastomoses. The donor had a normal 12-lead ECG and no apparent history of arrhythmias. The total transplant "ishaemic time" was $185 \mathrm{~min}$ utes. Early postoperative progress was uncomplicated.

Holter monitoring performed five weeks postoperatively revealed evidence of sinus node dysfunction in the absence of symptoms, with a nocturnal sinus bradycardia of 40 beats $/ \mathrm{min}$. Several episodes of myocardial cellular rejection necessitated a change in immunosuppression treatment from cyclosporin to tacrolimus (FK 506). During one episode of rejection electrical cardioversion for atrial flutter was required. Subsequently, atrial flutter recurred in the absence of histological evidence of cellular rejection, requiring repeated electrical cardioversion and the commencement of antiarrhythmic treatment with amiodarone (fig $1)$.

Following the third cardioversion, a prolonged period of asystole prompted transient temporary pacemaker implantation. Initial consideration was given to continued amiodarone treatment together with atrial or dual chamber permanent pacing. In view of further episodes of atrial flutter, electrophysiological evaluation was performed that demonstrated a macroflutter circuit close to the recipientdonor right atrial anastomosis. This pathway was successfully ablated with one burn, resulting in the attainment of donor heart sinus rhythm at 65 beats $/ \mathrm{min}$. Atrial flutter could not be induced by right atrial stimulation or isoprenaline infusion. 


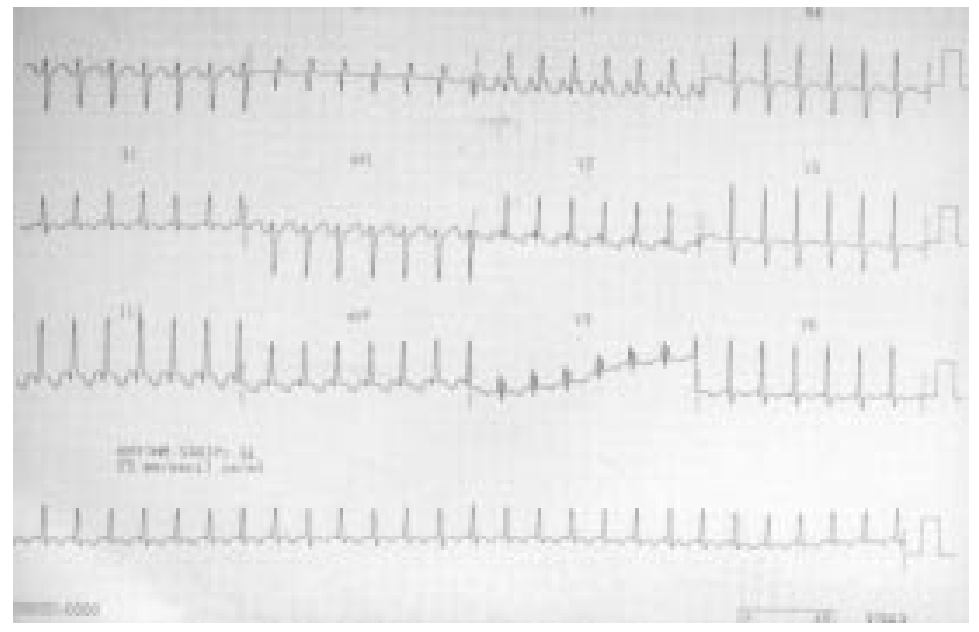

Figure 1 ECG showing atrial flutter with ventricular rate of 150 beats/min.

Subsequently, the patient was able to conduct normal activities without symptoms, and her chronotropic response at formal exercise testing revealed an adequate tachycardic response. Nevertheless, 10 weeks following ablation and cessation of amiodarone, presyncopal symptoms were found, associated with daytime and nocturnal sinus pauses of 2.9 seconds and 4.2 seconds, respectively. There was no evidence of AV node conduction impairment. In view of the continued absence of cellular rejection and the evident denervated state of the heart, the pauses reflected significant donor sinus node dysfunction; therefore, an AAIR permanent pacing system was implanted. At the time of pacemaker implantation atrial pacing at 150 beats/min did not produce significant PR interval prolongation or AV block.

\section{Discussion}

The isolation of the anatomical substrate of reentry in atrial flutter has allowed radiofrequency ablation to be employed to interrupt the arrhythmia circuit. In common flutter the preferred area for ablation is the isthmus between the inferior vena cava and tricuspid valve. Success rates have been quoted at approximately $90 \%$, with recurrent atrial flutter occurring in $10-46 \%$ of cases. Atrial fibrillation may appear in approximately $30 \%$ of cases. $^{2}$
Atrial flutter occurring in the presence of atrial scars following atrial baffle surgery or the Fontan procedure has been successfully treated with radiofrequency ablation. ${ }^{7}$ Radiofrequency ablation procedures following heart transplantation have previously been described. ${ }^{6}$ Donor hearts with occult bypass tracts have been identified post-transplantation with ablation techniques employed for Wolff-ParkinsonWhite syndrome. ${ }^{8}$ Right sided radiofrequency ablation has been reported in native heart atrial flutter/fibrillation in a heterotopic heart transplant recipient. ${ }^{9}$

The present case demonstrated the efficacy of radiofrequency ablation for atrial flutter following orthotopic heart transplantation. Careful surveillance allowed the identification of sinus node dysfunction, necessitating permanent atrial pacing. Associated sinus node dysfunction has been reported in inducible atrial flutter following orthotopic heart transplantation. ${ }^{10}$ Continued surveillance will be necessary to identify recurrent atrial arrhythmias.

1 Crijns HJG, Van Gelder IC, Tieleman RG, Brugemann J, De Kam PJ, Gosselink ATM, et al. Long-term outcome of electrical cardioversion in patients with chronic atrial flutter. Heart 1996;77:56-61.

2 Cosio FG, Arribas F, Lopez-Gil M, Gonzalez HD. Atrial flutter mapping and ablation II. Radiofrequency ablation of atrial flutter circuits. $P A C E$ 1996;19:965-75.

3 Scott CD, Dark JH, McComb JM. Arrhythmias after cardiac transplantation. Am $\mathcal{F}$ Cardiol 1992;70:1061-3.

4 Jacquet L, Ziady G, Stein K, Griffith B, Armitage J, Hardesty R, et al. Cardiac rhythm disturbances early after orthotopic heart transplantation: prevalence and clinical importance of the observed abnormalities. $\mathcal{F} \mathrm{Am}$ Coll Cardiol 1990;16:832-7.

5 Grant SC, Khan MA, Faragher EB, Yonan N, Brooks NH. Atrial arrhythmias and pacing after orthotopic heart transplantation: bicaval versus standard atrial anastomosis. Br Heart $\mathcal{F}$ 1995; 74:149-53.

6 Li YG, Gronefeld G, Hohnloser SH. Radiofrequency catheter ablation of atrial flutter after orthotopic heart transplantation. F Cardiovasc Electrophysiol 1996;7:108690.

7 Balaji S, Johnson TB, Sade RM, Case CL, Gillette PC. Management of atrial flutter after the Fontan procedure. $\mathcal{F}$ Am Coll Cardiol 1994;23:1209-15.

8 Rothman SA, Hsia HH, Bove AA, Jeevanandam V, Miller JM. Radiofrequency ablation of Wolff-Parkinson-White syndrome in a donor heart after orthotopic heart transplantation. f Heart Lung Transpl 1994;13:905-9.

9 Fenelon G, Goethals M, Brugada P. Two hearts beating as one: radiofrequency ablation of the His bundle in a heterotopic heart transplant patient. PACE 1996;19:374-5.

10 Heinz G, Hirschl MM, Kratochwill C, Buxbaum P, Laufer $\mathrm{G}$, Gossinger $\mathrm{H}$, et al. Inducible atrial flutter and fibrillation after orthotopic heart transplantation. F Heart Lung Transpl 1993;12:517-21. 